\title{
Comparison of Laservibrometers and Strain Gauges as Reference Sensors for High-g Shock Accelerometer Calibration Systems
}

\author{
Martin Brucke ${ }^{1}$, Georg Siegmund ${ }^{2}$, Christian Ehrmann ${ }^{2}$, Uwe Bühn $^{1}, \underline{\text { Michael Mende }}{ }^{l}$ \\ ${ }^{1}$ SPEKTRA Schwingungstechnik und Akustik GmbH, Dresden, Germany, sales@spektra-dresden.de \\ ${ }^{2}$ Polytec GmbH, Waldbronn, Germany, G.Siegmund@polytec.de
}

\begin{abstract}
: gauges) be verified? systems.

\section{INTRODUCTION}

In recent years SPEKTRA Schwingungstechnik und Akustik GmbH Dresden has had more and more customers who asked for shock exciters with an amplitude range above $100000 \mathrm{~m} / \mathrm{s}^{2}$. For some of their applications they needed $2000000 \mathrm{~m} / \mathrm{s}^{2}$ or higher. Typical applications were on the one hand the calibration of high-shock accelerometers used e.g. in the aerospace industry or in military applications. On the other hand testing applications like endurance testing of MEMS (Micro-ElectroMechanical Systems) structures, failure investigations of MEMS structures (sticking effects), investigations of contact forces between surfaces and particles or measurements of shock waves in medical applications also required the use of high shock exciters.
\end{abstract}

Applications that require measurements of shock type acceleration in an amplitude range above $100000 \mathrm{~m} / \mathrm{s}^{2}$ are becoming increasingly more important in the industry. Thus also an accurate calibration of accelerometers used for such measurements is compulsory. This paper focuses on appropriate reference standards and the influence variables that determine the measurement uncertainty of high shock calibration systems using Hopkinson-bar shock exciters up to amplitudes of $2000000 \mathrm{~m} / \mathrm{s}^{2}$. What are the main variables that determine the measurement uncertainty? How can the uncertainty contributions of different reference sensors (laser vibrometer and strain

The paper aims to give an overview and also tries to identify where future research will be necessary to understand influence variables and optimize the measurement uncertainty of such high-shock calibration

Hopkinson-bars turned out to be the most reliable and feasible exciters for such applications. Using equitation (1) (see also [1]) where $\mathrm{c}_{0}$ is the velocity of sound in the bar material, E its Young's modulus and $\mathrm{A}$ the cross section area of the bar, it was clear from a theoretical point of view that it is possible to generate very high acceleration amplitudes. $a(t)=\frac{2 c_{0}}{E \cdot A} \frac{d F(t)}{d t}$

In practice shock amplitudes up to $2000000 \mathrm{~m} / \mathrm{s}^{2}$ can be generated reliably today and first results indicate that even $5000000 \mathrm{~m} / \mathrm{s}^{2}$ could already be realized with an experimental setup. The duration of the generated shocks were typically shorter than $50 \mu$ s for the complete 'acceleration dipole cycle'.

But how can we measure such high acceleration amplitudes accurately? What are appropriate reference standards for such measurements and is it possible to trace back such a reference to a national standard? These were the questions that came up when the first systems were build. The authors mainly used two types of reference sensors:
a. Strain gauges
b. Laser vibrometers

\section{STRAIN GAUGES}

The theory of the Hopkinson-bar indicates that strain gauges can be used as reference sensors since the acceleration output of the bar is proportional to the time derivation of the strain caused by the compression wave travelling through the bar (2). 


$$
a(t)=2 c_{0} * \frac{d \varepsilon(t)}{d t}
$$

So a calibrated strain gauge seems to be an inexpensive reference sensor. Fig. 1 shows a typical setup for measurements with a Hopkinson-bar:

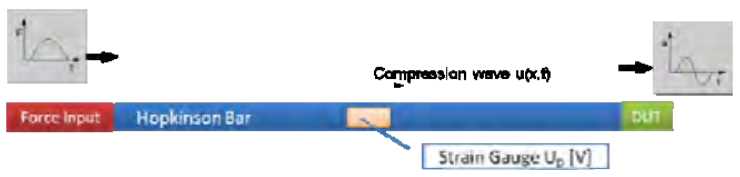

Fig. 1: Using a strain gauge as reference sensor $(D U T=$ device under test $)$

The following equations apply for this setup, where

- $\mathrm{c}_{0}=4830 \mathrm{~m} / \mathrm{s}$ is the speed of sound in Titan

- $\mathrm{K}_{\mathrm{DMS}}=2.1$ is the sensitivity of the strain gauge

- $\mathrm{U}_{\mathrm{S}}$ voltage applied to the strain gauge half bridge

$v(t)=2 \cdot \epsilon_{0} \cdot g(t)$

$U_{D}(t)=\frac{U_{S}}{2} \cdot K_{D M S} \cdot s(t)$

After inserting (3) in (4) a theoretical 'velocity sensitivity' of the strain gauge can be calculated that can in turn be used to calculate $a(t)$ from $U_{D}(t)$

$$
\frac{U_{D}(t)}{v(t)}=\frac{U_{S} \cdot K_{D W S}}{4 \cdot \epsilon_{0}}=0,00217\left[\frac{V^{2}}{\frac{m}{s}}\right]
$$

For $\mathrm{K}_{\mathrm{DMS}}$ the manufacturer of the strain gauges gives a relatively low measurement uncertainty of $\pm 0.5 \%$. But how can the speed of sound in the bar material be determined? If material parameters like the Young's modulus $E$ of the material and its density $\rho$ are used, $c_{0}$ can be derived from

$$
c_{0}=\sqrt{\frac{E}{\rho}}
$$

But $E$ is determined with a typical $8 \%$ measurement uncertainty from static tensile tests. What does this say about highly dynamic processes in the material as can be observed in Hopkinson-bars? Thus although calibrated strain gauges are available on the market, it is obvious that a several influence variables like mounting of the gauges, material parameters or dispersion of the waves in the bar can lead to high contributions to the measurement uncertainty.

Optical measurement methods avoid such problems. So a laser vibrometer was used as 'trusted reference standard' in order to investigate the variables that influence the measurement uncertainty of strain gauges as reference sensors. By means of a calibration setup (see Fig. 2) with a laser vibrometer as reference sensor and strain gauges as DUT, the 'velocity sensitivity' of the strain gauges was measured and compared with the calculated value.

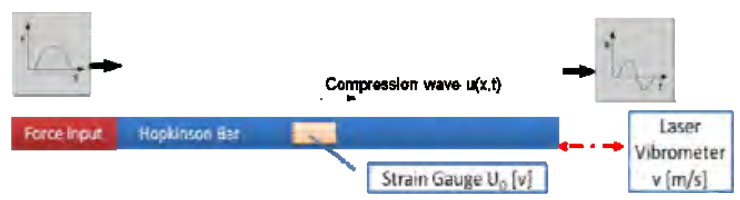

Fig. 2: Transfer calibration of a strain gauge using a laser vibrometer as transfer standard

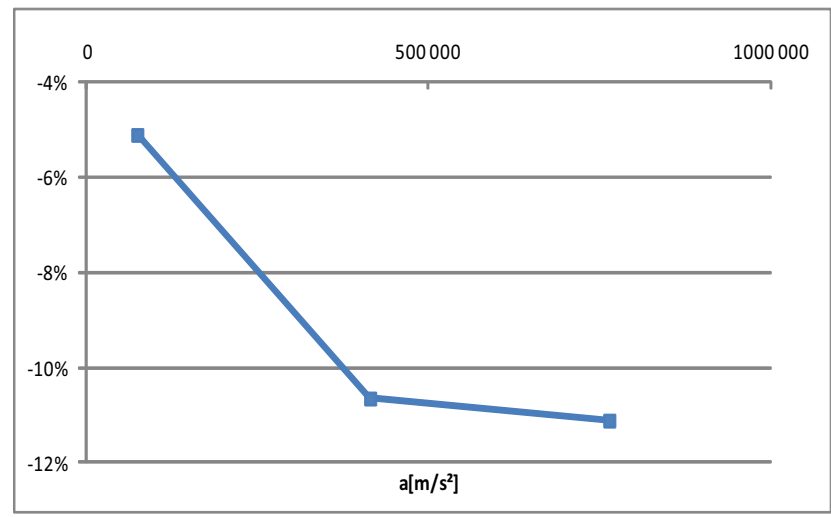

Fig. 3: Deviation of the measured 'velocity sensitivity' of the strain gauges compared to the calculated nominal value

The results (see Fig. 3) showed significant deviations at lower amplitudes that increased up to $11 \%$ at amplitudes around $800000 \mathrm{~m} / \mathrm{s}^{2}$. An explanation for this increasing deviation can be found if we look at the time response of laser vibrometer and strain gauges (see Fig. 4). The time shift between both signals is related to the mounting position of the strain gauges in the middle of the bar. But looking at the shape of the signals, both show quite similar shapes at moderate amplitudes (here $80000 \mathrm{~m} / \mathrm{s}^{2}$ ), while the signals differ significantly at ten times higher amplitudes. This is caused by dispersion of the waves in the bar.

However, the results above show that the nominal calculated 'velocity sensitivity' of the strain gauges can hardly be used as a reference if the assumption is correct that the laser vibrometer is measuring more accurately than the strain gauges. In the next section it will be shown that this assumption is justified and thus a calibration of the strain gauges with a laser vibrometer at $100000 \mathrm{~m} / \mathrm{s}^{2}$ is currently the standard method at SPEKTRA to calibrate strain gauges as reference standards for Hopkinson-bars. This calibration at one amplitude level accepts that the increase of the deviation of the determined 'velocity sensitivity' compared to the laser vibrometer sensitivity will not be corrected. Future versions of the CS18 calibration software will be able to cope with the dispersion effects at high amplitudes. 

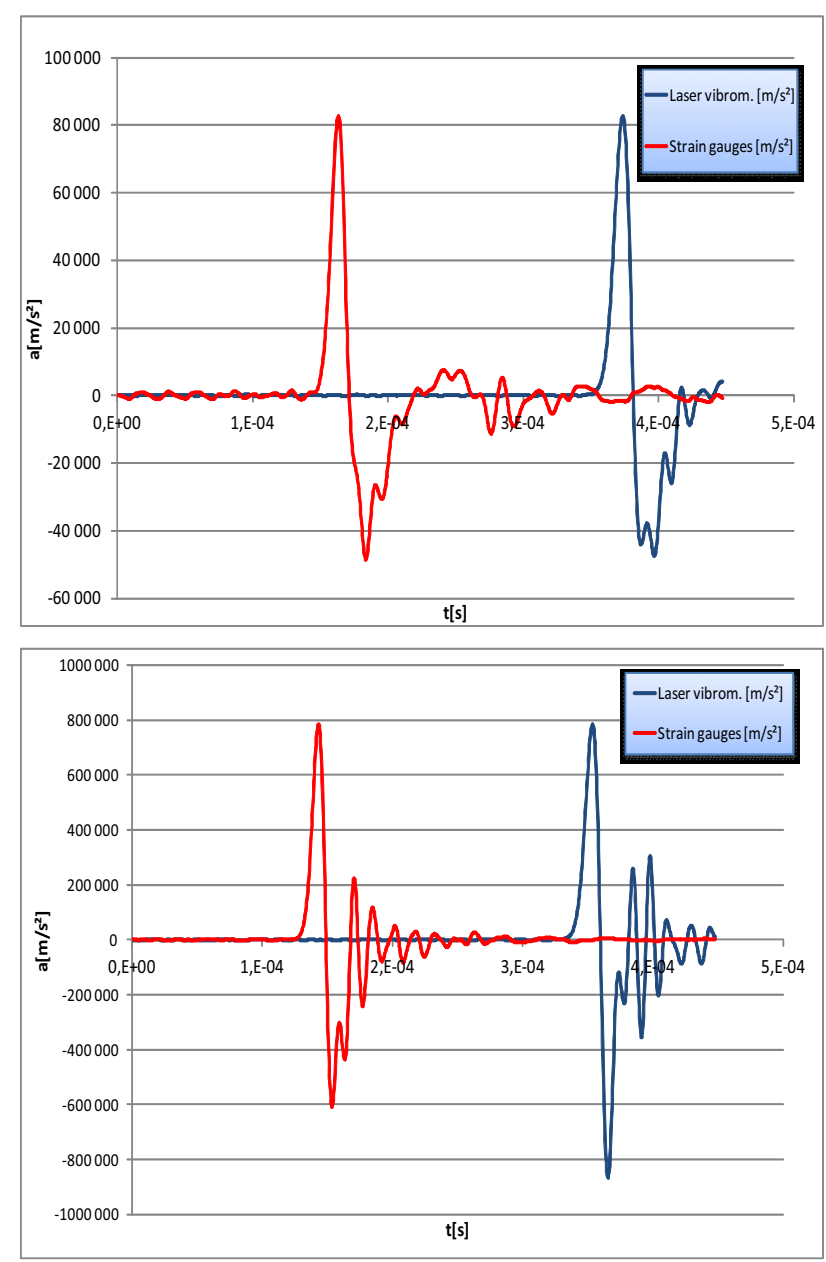

Fig. 4: The time response of laser vibrometer and strain gauges shows significant differences at high shock amplitudes

Fig. 5 shows the measured deviation of the sensitivity of a laser vibrometer compared to strain gauges used as a reference sensor. The strain gauge was calibrated as described before. The measurement uncertainty budget that could be obtained for this system shows a good measurement uncertainty of $3 \%$ to $6 \%$ in the amplitude range $200000 \mathrm{~m} / \mathrm{s}^{2}$ to $1000000 \mathrm{~m} / \mathrm{s}^{2}$. Above this range the uncertainty increases up to $8 \%$. All current measurements seem to verify this uncertainty budget.

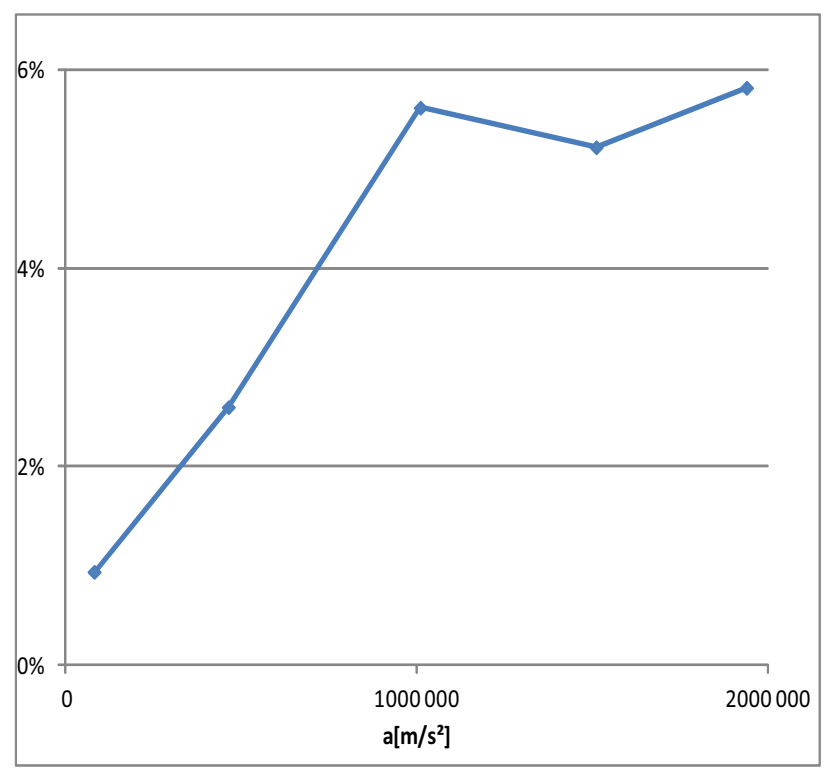

Fig. 5: Deviation of the measured 'velocity sensitivity' of the laser vibrometer compared to the nominal value. The reference strain gauges were calibrated at $100000 \mathrm{~m} / \mathrm{s}^{2}$

\section{Laser vibrometer}

As mentioned above Laser Doppler Vibrometers (LDV) were used as trusted reference sensors for the calibration of strain gauges. But as was shown in [4], a LDV with analog velocity decoder can have significant velocity errors at the limits of the working range. Although modern LDVs with digital velocity decoders were used for these measurements, it was not absolutely clear if similar problems could occur with these devices as well. So at the beginning of their work the authors used two different types of Polytec laser vibrometers [OFV-5000 with OFV-505 optic $(10 \mathrm{~m} / \mathrm{s})$ and an OFV-5000-S with an OFV552 fiber optic $(20 \mathrm{~m} / \mathrm{s})$ ] with different optics, different amplitude ranges and different velocity decoders to get a feeling for how far the results can be trusted.

A calibration setup was chosen for measurements where one laser vibrometer served as reference sensor and the other vibrometer as DUT. Measurements were performed at different acceleration levels up to $850000 \mathrm{~m} / \mathrm{s}^{2}$. At each acceleration level the measurements were repeated five times. In Fig. 6 it can be seen that the time signals of both vibrometers were almost identical. Further analysis showed that the measured sensitivity deviation compared to the nominal sensitivity of the DUT vibrometer was very small at all acceleration amplitudes. However, more investigations were performed at Polytec in order to fortify the results above by means of electrical measurements bypassing the linear optical system and stressing the electronics and data processing part of the vibrometer. 


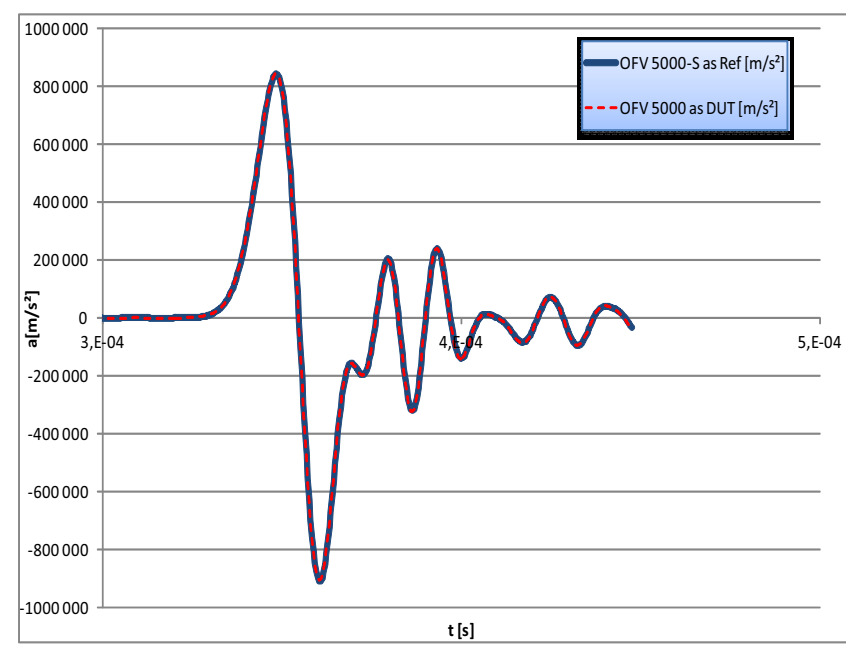

Fig. 6: Time signals of two different laser vibrometer measuring a $850000 \mathrm{~m} / \mathrm{s}^{2}$ shock

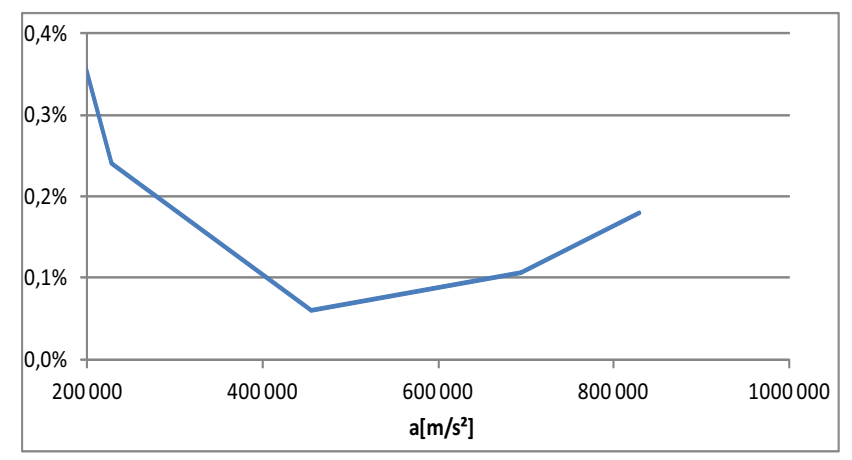

Fig. 7: Deviation of the measured sensitivity of the OFV5000 laser vibrometer compared to the nominal sensitivity (an OFV-5000-S was used as reference sensor)

For primary calibration of shock transducers according to ISO 16063-13, the reference quantity must be acquired by means of laser interferometry. The principle of laser interferometry is incorporated in national measurement standards as well as in commercially available LDVs. Laser vibrometers with digital signal processing, in particular, can serve as reference standards in primary calibration systems [3]. Due to their non-contact working principle, which uses photons as a carrier of measurement information, LDVs can acquire motions in a much wider amplitude and frequency range than conventional transducers. Their internal optical interferometer performs a linear transformation of the motion quantities displacement and velocity into phase and frequency modulation of an electrical signal. Thereby, the wavelength $\lambda$ of the laser acts as a material measure. For the modulation parameters $\Delta f$ and $\Delta \varphi$ and the related motion quantities $\hat{v}$ and $\hat{s}$ the simple relationships given in equations (7) and (8) apply.

$$
\begin{aligned}
\Delta f & =\frac{2 \theta}{A} \\
\Delta \varphi & =\frac{4 \pi \cdot \xi}{A}
\end{aligned}
$$

Up to this point, the measurement principle has no relevant upper limits for the motion quantities velocity, acceleration or vibration frequency due to the physical properties of light. Only object displacement is limited to a few meters by the optical depth of field. Due to these advantages the principle of laser interferometry is ideally suited for the acquisition of shock events with extreme acceleration amplitudes. However, limits are set by real technical properties of subsequent signal processing, starting with the photo detector. In the case of a heterodyne LDV, first of all, the center frequency has to be higher than the peak frequency deviation according to equation (7). In order to transmit a peak velocity of $20 \mathrm{~m} / \mathrm{s}$, corresponding to a frequency deviation of $63.2 \mathrm{MHz}$ at $\lambda=632 \mathrm{~nm}$, the center frequency should be at least $70 \mathrm{MHz}$. The operating bandwidth of the photo detector and the subsequent signal processing blocks must be at least twice the center frequency in order to prevent nonlinear distortions. Detailed bandwidth requirements for reference laser interferometers are given in the respective ISO standards, e.g. [3]. Within their specified amplitude limits, properly designed optical sections of commercial LDVs, including the photo detectors, have negligible impact on the modulation content of broadband Doppler signals as present when acquiring high-shock events.

The LDV Polytec OFV-5000-S with a center frequency of $80 \mathrm{MHz}$ covers a velocity amplitude range of $\pm 20 \mathrm{~m} / \mathrm{s}$. Its full-scale frequency range of DC to $1.5 \mathrm{MHz}$ allows for a peak acceleration of $1.88 \cdot 10^{8} \mathrm{~m} / \mathrm{s}^{2}$. Due to a lack of suitable shock exciters this limit has not yet been proven in practice. In 1995, for the first time a complete dynamic certification of a Polytec LDV was performed at Sandia National Laboratories, Albuquerque [4]. The investigation was based on precisely synthesized heterodyne Doppler signals, modulating a laser diode whose output again illuminated the photo detector of the LDV. It was shown that even this old LDV with analog demodulator could fairly handle shock impulses with peak velocities of $10 \mathrm{~m} / \mathrm{s}$ and peak accelerations of $0.94 \cdot 10^{8} \mathrm{~m} / \mathrm{s}$. However, peak velocity errors up to $10 \%$ due to ringing and overshot were observed when approaching the operating range limits of the LDV.

In the following we will demonstrate the capabilities of the digital OFV-5000-S digital laser vibrometer with respect to high-shock acquisition, using a similar method as reported in [4]. Since no light emitting diode with trusted modulation frequency response was available, simulated Doppler signals were fed directly into the RF input of the LDV controller, bypassing the photo detector. This simplification is justified, since the photo detector of the dedicated optical sensor is trusted to fulfill the bandwidth requirements discussed above by far. 
More detailed analyses of error sources in laser vibrometry were conducted in $[5,6]$.
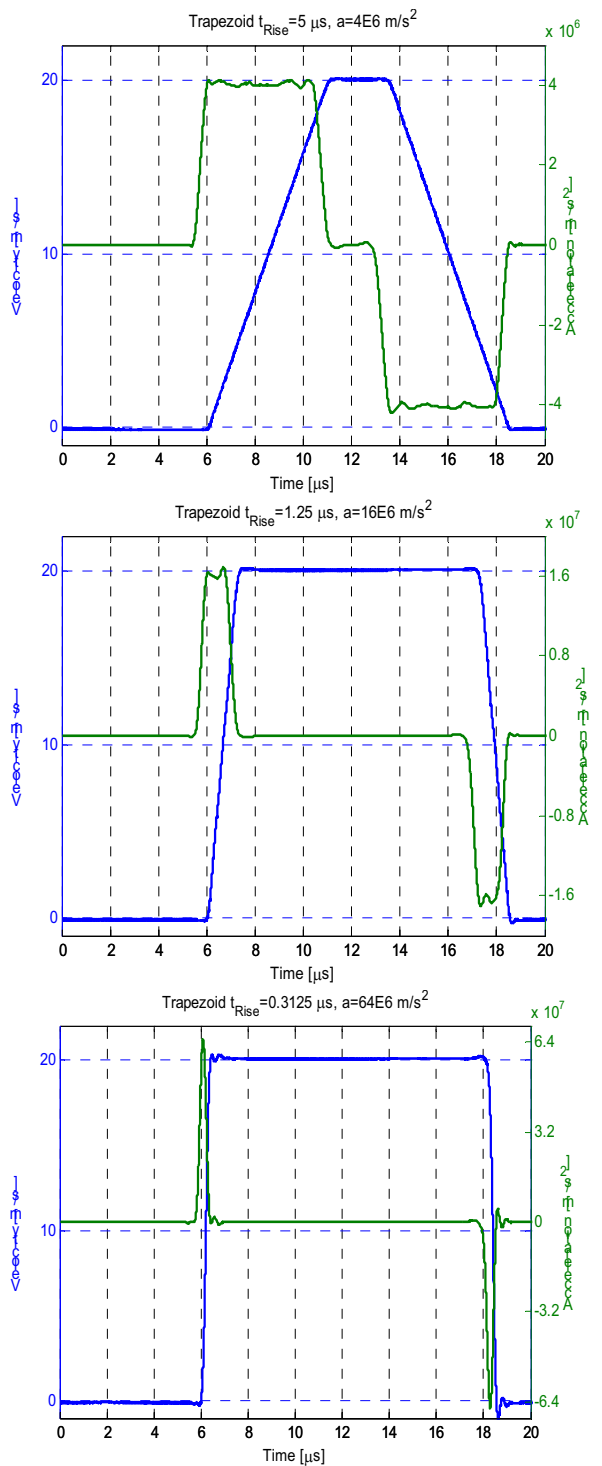

Fig. 8: Measured trapezoidal full-scale velocity impulses with different rise times

For demonstration of both amplitude accuracy and linearity of the OFV-5000-S with digital VD-09-S velocity decoder, Doppler signals representing trapezoidal velocity impulses with $20 \mathrm{~m} / \mathrm{s}$ peak amplitude and varying rise time were simulated based on the relationship given in equation (7). Waveform data were fed into a digital function generator which generated the corresponding modulated RF signal for input to the vibrometer controller. Fig. 8 shows three velocity output waveforms with rise times of 5.0, 1.25 and 0.3125 $\mu \mathrm{s}$ (zero to peak), corresponding to acceleration amplitudes of $4 \cdot 10^{6} \mathrm{~m} / \mathrm{s}^{2}, \quad 1.6 \cdot 10^{7} \mathrm{~m} / \mathrm{s}^{2}$ and $6.4 \cdot 10^{7} \mathrm{~m} / \mathrm{s}^{2}$. Ringing only occurs at the shortest rise time, where the acceleration is about one third of the specified limit but higher than required for shock calibration. Velocity error without ringing is lower than the resolution of the digital oscilloscope used.
The corresponding acceleration time histories were calculated by numerical off-line differentiation and subsequent averaging of 16 pulses.

Another set of experiments was conducted the same way but using Gaussian velocity impulses with shapes similar to those observed at the far end of a Hopkinson-bar. Fig. 9 shows measured velocity and calculated acceleration waveforms with different pulse widths. The peak acceleration values obtained by differentiation of low-pass filtered velocity data are very close to the theoretical peak values resulting from the Gaussian velocity impulse parameters as to be seen in

Table 1.

Table 1: Results with synthetic Gaussian velocity impulses

\begin{tabular}{|c|c|c|c|c|}
\hline $\begin{array}{c}\text { Pulse } \\
\text { Width } \\
\text { (full } \\
\text { cycle) }\end{array}$ & $\begin{array}{c}\text { Pulse Width } \\
@ 0.606 v_{\text {peak }}\end{array}$ & $\begin{array}{c}\text { Source } \\
\text { Acceleration } \\
\text { (peak) }\end{array}$ & $\begin{array}{c}\text { Output } \\
\text { Acceleration } \\
\text { (peak) }\end{array}$ & $\begin{array}{c}\text { Relative } \\
\text { Error }\end{array}$ \\
\hline $80 \mu \mathrm{s}$ & $26.4 \mu \mathrm{s}$ & $0.886 \cdot 10^{6} \mathrm{~m} / \mathrm{s}^{2}$ & $0.90 \cdot 10^{6} \mathrm{~m} / \mathrm{s}^{2}$ & $1.6 \%$ \\
\hline $20 \mu \mathrm{s}$ & $6.6 \mu \mathrm{s}$ & $3.54 \cdot 10^{6} \mathrm{~m} / \mathrm{s}^{2}$ & $3.59 \cdot 10^{6} \mathrm{~m} / \mathrm{s}^{2}$ & $1.4 \%$ \\
\hline $5 \mu \mathrm{s}$ & $1.65 \mu \mathrm{s}$ & $1.42 \cdot 10^{7} \mathrm{~m} / \mathrm{s}^{2}$ & $1.44 \cdot 10^{7} \mathrm{~m} / \mathrm{s}^{2}$ & $1.4 \%$ \\
\hline
\end{tabular}

The good agreement of both velocity and displacement peak output values with the simulated pulse parameters verifies the ability of the laser vibrometer to acquire impulses that are typical for a Hopkinson-bar high-shock machine.

Since some of the main influence variables like mounting of the strain gauges or dispersion are not applicable for reference laser vibrometers, the measurement uncertainty of such primary calibration systems is expected to stay below $3 \%$ even at amplitudes higher than $1000000 \mathrm{~m} / \mathrm{s}^{2}$ and to drop below $2 \%$ at amplitudes less than $100000 \mathrm{~m} / \mathrm{s}^{2}$. 

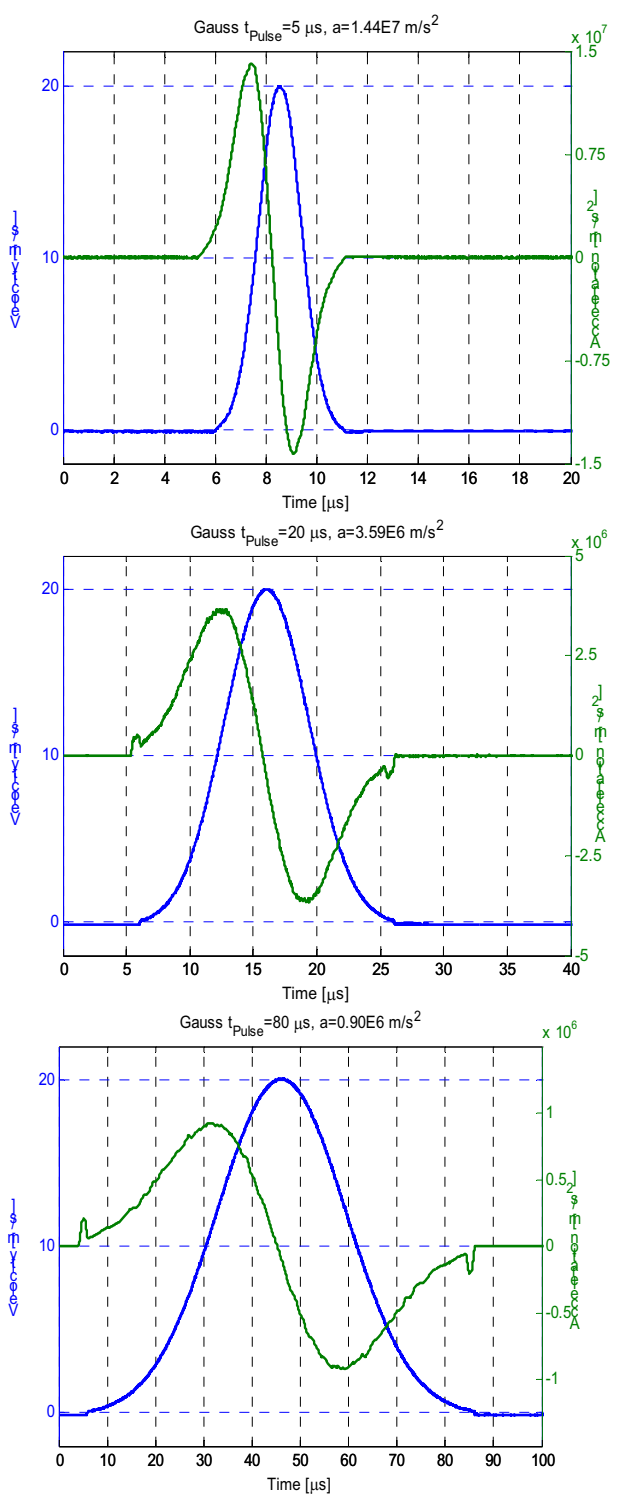

Fig. 9: Measured Gaussian velocity and acceleration impulses with pulse widths 5, 20 and $80 \mu \mathrm{s}$

\section{Calibration of a High-Shock Sensor}

In order to check the results of the measurements above with a real-world DUT, a high-shock accelerometer (Endevco 7270A-200K) with an extreme measurement range up to $2000000 \mathrm{~m} / \mathrm{s}^{2}$ was calibrated with an OFV-5000 laser vibrometer as well as with a calibrated strain gauge as reference on the Hopkinson-bar. This type of accelerometer has a very high resonance frequency (above $1 \mathrm{MHz}$ ) and can be expected to measure independent from the duration of the shocks and quite linear up to $500000 \mathrm{~m} / \mathrm{s}^{2}$ (fourth of the measurement range). Thus it made sense to determine a reference sensitivity of the accelerometer on a shock pendulum at $2000 \mathrm{~m} / \mathrm{s}^{2}$ traceable to PTB and compare it to the sensitivity measured on the Hopkinson-bar. Fig. 10: shows the results of these measurements. The determined sensitivity values were quite close to the reference value independent from the reference sensor. So also these calibrations confirm the assumption of a measurement uncertainty in this amplitude range of below 3\% to $6 \%$ with a strain gauge and below $2 \%$ with a LDV as reference sensor. In fact the data indicate that the measurement uncertainty may be even lower.

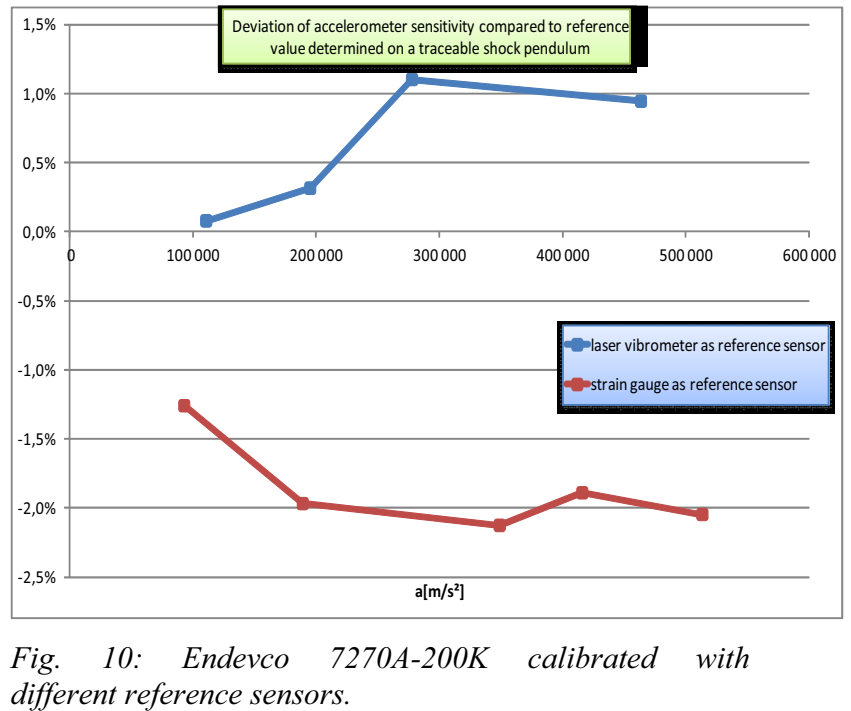

different reference sensors.

\section{Conclusions}

Strain gauges as well as laser vibrometers are appropriate reference sensors for shock calibration and test systems up to acceleration amplitudes of $2000000 \mathrm{~m} / \mathrm{s}^{2}$. The laser vibrometer with its noncontact measurement offers a much higher accuracy than the electro-mechanical measurement with strain gauges. Our measurements have shown that the more or less static factory calibration of the strain gauges, mounting, and dispersion of the compression waves lead to a quite high measurement uncertainty that increases with the amplitude. A transfer calibration of the strain gauges mounted on the Hopkinson-bar allows decreasing the measurement uncertainty significantly. In the future it may also be possible to cope with uncertainties caused by dispersion.

However, for both, the usage of a laser vibrometer as a transfer standard for the calibration of strain gauges as well as for the integration into a primary calibration system, a calibrated laser vibrometer is necessary. A traceable calibration of laser vibrometers using shock exciters that covers acceleration amplitudes up the maximum amplitudes described above would be desirable but is currently not provided by any National Metrology Institute. Therefore research should be performed in order to improve the measurement uncertainty of high-shock measurement systems and thus to cover the current and future needs of the industry.

[1] M. Brucke, „Alternative Methoden zur Anregung von Wellen in Stäben zur Kalibrierung von Beschleunigungsaufnehmern“, Dissertation TU Braunschweig; 2009 
[2] International Standard ISO 16063-13 - Methods for the calibration of vibration and shock transducers Part 13: Primary shock calibration using laser interferometry, Int. Org. for Standardization (ISO), Geneva (2001)

[3] International Standard ISO 16063-41 "Methods for the calibration of vibration and shock transducers - Part 41: Calibration of laser vibrometers", Int. Org. for Standardization (ISO), Geneva (2011)

[4] V. I. Bateman, B. D. Hansche, O. M. Solomon, "Use of Laser Doppler Vibrometers for High Frequency Accelerometer Characterizations", 66. Shock and vibration symposium, Biloxi (US), 1995.

[5] G. Siegmund, "Sources of measurement error in laser Doppler vibrometers and proposal for unified specifications", 8th International Conference on Vibration Measurements by Laser Techniques, Proc. of SPIE Vol. 7098, 2008

[6] M. Winter, H. Füser, M. Bieler, G. Siegmund, C. Rembe, "The Problem of Calibrating Laser-Doppler Vibrometers at High Frequencies", $10^{\text {th }}$ International Conference on Vibration Measurements by Laser and Noncontact Techniques, Ancona, 2012. 